Groups Geom. Dyn. 4 (2010), 419-432

DOI $10.4171 / \mathrm{GGD} / 89$
Groups, Geometry, and Dynamics

(C) European Mathematical Society

\title{
The ergodic theory of free group actions: entropy and the $f$-invariant
}

\author{
Lewis Bowen
}

\begin{abstract}
Previous work introduced two measure-conjugacy invariants: the $f$-invariant (for actions of free groups) and $\Sigma$-entropy (for actions of sofic groups). The purpose of this paper is to show that the $f$-invariant is essentially a special case of $\Sigma$-entropy. There are two applications: the $f$-invariant is invariant under group automorphisms and there is a uniform lower bound on the $f$-invariant of a factor in terms of the original system.
\end{abstract}

Mathematics Subject Classification (2010). 37A35.

Keywords. Free groups, entropy, $f$-invariant.

\section{Introduction}

The paper [Bo08b] introduced a measure-conjugacy invariant, called $\Sigma$-entropy, for measure-preserving actions of a sofic group. This was applied, for example, to classify Bernoulli shifts over an arbitrary countable linear group. Previously, [Bo08a] introduced the $f$-invariant for measure-preserving actions of free groups. The invariants of both papers have strong analogies with classical Kolmogorov-Sinai entropy. The purpose of this paper is to show that the $f$-invariant is essentially a special case of $\Sigma$-entropy. We apply this result to show the $f$-invariant does not change under group automorphisms and that there is a lower bound on the $f$-invariant of a factor in terms of the $f$-invariant of the system. The introductions to [Bo08a]-[Bo08b] provide further background and motivation for $\Sigma$-entropy and the $f$-invariant.

To define $\Sigma$-entropy precisely, let $G$ be a countable group and let $\Sigma=\left\{\sigma_{i}\right\}_{i=1}^{\infty}$ be a sequence of homomorphisms $\sigma_{i}: G \rightarrow \operatorname{Sym}\left(m_{i}\right)$ where $\operatorname{Sym}\left(m_{i}\right)$ denotes the full symmetric group of the set $\left\{1, \ldots, m_{i}\right\} . \Sigma$ is asymptotically free if

$$
\lim _{i \rightarrow \infty} \frac{\left|\left\{1 \leq j \leq m_{i} \mid \sigma_{i}\left(g_{1}\right) j=\sigma_{i}\left(g_{2}\right) j\right\}\right|}{m_{i}}=0 .
$$

for every pair $g_{1}, g_{2} \in G$ with $g_{1} \neq g_{2}$. The treatment of $\Sigma$-entropy given next differs from [Bo08b] in two respects: for simplicity, we assume that each $\sigma_{i}$ is a homomorphism and we use observables rather than partitions to define it. 
We will write $G \curvearrowright^{T}(X, \mu)$ to mean $(X, \mu)$ is a standard probability measure space and $T=\left(T_{g}\right)_{g \in G}$ is an action of $G$ on $(X, \mu)$ by measure-preserving transformations. This means that for each $g \in G, T_{g}: X \rightarrow X$ is a measure-preserving transformation and $T_{g_{1}} T_{g_{2}}=T_{g_{1} g_{2}}$. An observable of $(X, \mu)$ is a measurable map $\phi: X \rightarrow A$ where $A$ is a finite or countably infinite set. We will say that $\phi$ is finite if $A$ is finite. Roughly speaking, the $\Sigma$-entropy rate of $\phi$ is the exponential rate of growth of the number of observables $\psi:\left\{1, \ldots, m_{i}\right\} \rightarrow A$ that approximate $\phi$. In order to make precise what it means to approximate, we need to introduce some definitions.

If $\phi: X \rightarrow A$ and $\psi: X \rightarrow B$ are two observables, then the join of $\phi$ and $\psi$ is the observable $\phi \vee \psi: X \rightarrow A \times B$ defined by $\phi \vee \psi(x)=(\phi(x), \psi(x))$. If $g \in G$ then $T_{g} \phi: X \rightarrow A$ is defined by $T_{g} \phi(x)=\phi\left(T_{g} x\right)$. If $H \subset G$ is finite, then let $\phi^{H}:=\bigvee_{h \in H} T_{h} \phi . \phi^{H}$ maps $X$ into $A^{H}$, the direct product of $|H|$ copies of $A$. Let $\phi_{*}^{H} \mu$ denote the pushforward of $\mu$ on $A^{H}$. In other words, $\phi_{*}^{H}(\mu)(S)=\mu\left(\left(\phi^{H}\right)^{-1}(S)\right)$ for $S \subset A^{H}$.

For each $i$, let $\zeta_{i}$ denote the uniform probability measure on $\left\{1, \ldots, m_{i}\right\}$. If $\psi:\left\{1, \ldots, m_{i}\right\} \rightarrow A$ is an observable and $H \subset G$ then let $\psi^{H}:=\bigvee_{h \in H} \sigma_{i}(h) \psi$, where $\sigma_{i}(h) \psi:\left\{1, \ldots, m_{i}\right\} \rightarrow A$ is defined by $\sigma_{i}(h) \psi(j)=\psi\left(\sigma_{i}(h) j\right)$. Of course, $\psi^{H}$ depends on $\sigma_{i}$ but, to keep the notation simple, we will leave this dependence implicit. Let $\psi_{*}^{H} \zeta_{i}$ be the pushforward of $\zeta_{i}$ on $A^{H}$. Finally, let $d_{\sigma_{i}}^{H}(\phi, \psi)$ be the $l^{1}$-distance between $\phi_{*}^{H} \mu$ and $\psi_{*}^{H} \zeta_{i}$. In other words,

$$
d_{\sigma_{i}}^{H}(\phi, \psi)=\sum_{a \in A^{H}}\left|\phi_{*}^{H} \mu(a)-\psi_{*}^{H} \zeta_{i}(a)\right| .
$$

Definition 1. If $\phi: X \rightarrow A$ is an observable and $A$ is finite then define the $\Sigma$-entropy rate of $\phi$ by

$$
h(\Sigma, T, \phi):=\inf _{H \subset G} \inf _{\varepsilon>0} \limsup _{i \rightarrow \infty} \frac{1}{m_{i}} \log \left(\left|\left\{\psi:\left\{1, \ldots, m_{i}\right\} \rightarrow A \mid d_{\sigma_{i}}^{H}(\phi, \psi) \leq \varepsilon\right\}\right|\right) .
$$

The first infimum above is over all finite subsets $H \subset G$.

Definition 2. Define the entropy of $\phi$ by

$$
H(\phi):=-\sum_{a \in A} \mu\left(\phi^{-1}(a)\right) \log \left(\mu\left(\phi^{-1}(a)\right)\right) .
$$

Definition 3. If $\phi: X \rightarrow A$ is an observable and $A$ is countably infinite then let $\pi_{n}: A \rightarrow A_{n}$ be a sequence of maps such that

(1) $A_{n}$ is a finite set for all $n$;

(2) for each $i>j$ there is a map $\pi_{i j}: A_{i} \rightarrow A_{j}$ such that $\pi_{j}=\pi_{i j} \circ \pi_{i}$;

(3) $\pi_{n}$ is asymptotically injective in the sense that for all $a, b \in A$ with $a \neq b$ there exists $N$ such that $n>N$ implies $\pi_{n}(a) \neq \pi_{n}(b)$.

Now define

$$
h(\Sigma, T, \phi):=\lim _{n \rightarrow \infty} h\left(\Sigma, T, \pi_{n} \circ \phi\right) .
$$


In [Bo08b] it is proven that if $H(\phi)<\infty$ then this limit exists and is independent of the choice of sequence $\left\{\pi_{n}\right\}$.

An observable $\phi$ is generating if the smallest $G$-invariant $\sigma$-algebra on $X$ that contains $\left\{\phi^{-1}(a)\right\}_{a \in A}$ is equal to the $\sigma$-algebra of all measurable sets up to sets of measure zero. The next theorem is (part of) the main result of [Bo08b].

Theorem 1.1. Let $\Sigma=\left\{\sigma_{i}\right\}$ be an asymptotically free sequence of homomorphisms $\sigma_{i}: G \rightarrow \operatorname{Sym}\left(m_{i}\right)$ for a group $G$. Let $G \curvearrowright^{T}(X, \mu)$. If $\phi_{1}$ and $\phi_{2}$ are two finite-entropy generating observables then $h\left(\Sigma, T, \phi_{1}\right)=h\left(\Sigma, T, \phi_{2}\right)$.

This motivates the following definition.

Definition 4. If $\Sigma$ and $T$ are as above then the $\Sigma$-entropy of the action $T$ is defined by $h(\Sigma, T):=h(\Sigma, \phi)$, where $\phi$ is any finite-entropy generating observable (if one exists).

Next let us discuss a slight variation on $\Sigma$-entropy. Let $\left\{m_{i}\right\}_{i=1}^{\infty}$ be a sequence of natural numbers. For each $i \in \mathbb{N}$, let $\mu_{i}$ be a probability measure on the set of homomorphisms from $G$ to $\operatorname{Sym}\left(m_{i}\right)$. Let $\sigma_{i}: G \rightarrow \operatorname{Sym}\left(m_{i}\right)$ be chosen at random according to $\mu_{i}$. The sequence $\Sigma=\left\{\mu_{i}\right\}_{i=1}^{\infty}$ is said to be asymptotically free if for every pair $g_{1}, g_{2} \in G$ with $g_{1} \neq g_{2}$,

$$
\lim _{i \rightarrow \infty} \frac{\mathbb{E}\left[\left|\left\{1 \leq j \leq m_{i} \mid \sigma_{i}\left(g_{1}\right) j=\sigma_{i}\left(g_{2}\right) j\right\}\right|\right]}{m_{i}}=0
$$

where $\mathbb{E}[\cdot]$ denotes expected value. The $\Sigma$-entropy rate of an observable $\phi: X \rightarrow A$ with $A$ finite is defined by

$$
\begin{aligned}
h(\Sigma, T, \phi) & \\
& :=\inf _{H \subset G} \inf _{\varepsilon>0} \limsup _{i \rightarrow \infty} \frac{1}{m_{i}} \log \left(\mathbb{E}\left[\left|\left\{\psi:\left\{1, \ldots, m_{i}\right\} \rightarrow A \mid d_{\sigma_{i}}^{H}(\phi, \psi) \leq \varepsilon\right\}\right|\right]\right) .
\end{aligned}
$$

With these definitions in mind, Theorem 1.1 is still true if "homomorphisms" is replaced with "probability measures on the set of homorphisms".

Let us note one more generalization. If $G$ is a semigroup with identity then the above definitions still make sense. Using results from [Bo08c] it can be shown that Theorem 1.1 remains true.

Now let us recall the $f$-invariant from [Bo08a]. Let $G=\left\langle s_{1}, \ldots, s_{r}\right\rangle$ be either a free group or free semigroup of rank $r$. Let $G \curvearrowright^{T}(X, \mu)$. Let $\alpha$ be a partition of $X$ into at most countably many measurable sets. The entropy of $\alpha$ is defined by

$$
H(\alpha):=-\sum_{A \in \alpha} \mu(A) \log (\mu(A))
$$

where, by convention, $0 \log (0)=0$. If $\alpha$ and $\beta$ are partitions of $X$ then the join is the partition $\alpha \vee \beta:=\{A \cap B \mid A \in \alpha, B \in \beta\}$. Let $B(e, n)$ denote the ball of radius 
$n$ in $G$ with respect to the word metric induced by its generating set (which is either $\left\{s_{1}, \ldots, s_{r}\right\}$ if $G$ is a semigroup or $\left\{s_{1}^{ \pm 1}, \ldots, s_{r}^{ \pm 1}\right\}$ is $G$ is a group). Define

$$
\begin{aligned}
F(T, \alpha) & :=(1-2 r) H(\alpha)+\sum_{i=1}^{r} H\left(\alpha \vee T_{s_{i}}^{-1} \alpha\right), \\
\alpha^{n} & :=\bigvee_{g \in B(e, n)} T_{g}^{-1} \alpha, \\
f(T, \alpha) & :=\inf _{n} F\left(T, \alpha^{n}\right) .
\end{aligned}
$$

The partition $\alpha$ is generating if the smallest $G$-invariant $\sigma$-algebra containing $\alpha$ equals the $\sigma$-algebra of all measurable sets up to sets of measure zero.

Theorem 1.2. Let $G=\left\langle s_{1}, \ldots, s_{r}\right\rangle$ be a free group or free semigroup. Let $G \curvearrowright^{T}$ $(X, \mu)$. If $\alpha_{1}$ and $\alpha_{2}$ are two generating partitions with $H\left(\alpha_{1}\right)+H\left(\alpha_{2}\right)<\infty$ then $f\left(T, \alpha_{1}\right)=f\left(T, \alpha_{2}\right)$.

This theorem was proven in [Bo08c]. The special case in which $G$ is a group and $\alpha_{1}, \alpha_{2}$ are finite is the main result of [Bo08a]. Because of this theorem, we define the $f$-invariant of the action by $f(T):=f(T, \alpha)$, where $\alpha$ is any finite-entropy generating partition of $X$ (if one exists).

In order to relate this result with $\Sigma$-entropy, let us make the following definitions. If $\phi: X \rightarrow A$ is an observable, then let $\bar{\phi}=\left\{\phi^{-1}(a)\right\}_{a \in A}$ be the corresponding partition of $X$. Define $F(T, \phi):=F(T, \bar{\phi})$ and $f(T, \phi):=f(T, \bar{\phi})$. The main result of this paper is:

Theorem 1.3. Let $G=\left\langle s_{1}, \ldots, s_{r}\right\rangle$ be a free group or free semigroup of rank $r \geq 1$. Let $G \curvearrowright^{T}(X, \mu)$. Let $\phi$ be a finite observable. For $i \geq 1$, let $\mu_{i}$ be the uniform probability measure on the set of all homomorphisms from $G$ to $\operatorname{Sym}(i)$. Let $\Sigma=\left\{\mu_{i}\right\}_{i=1}^{\infty}$. Then $h(\Sigma, T, \phi)=f(T, \phi)$.

We will prove a refined version of this theorem as follows. Recall the definition of $d_{\sigma_{i}}^{H}(\phi, \psi)$ given above. Define

$$
d_{\sigma_{i}}^{*}(\phi, \psi):=\sum_{i=1}^{r} d_{\sigma_{i}}^{\left\{e, s_{i}\right\}}(\phi, \psi) .
$$

Theorem 1.4. Let $G$ and $T$ be as in the previous theorem. Let $\phi: X \rightarrow A$ be a finite observable. Let $\sigma_{i}: G \rightarrow \operatorname{Sym}(i)$ be a homomorphism chosen uniformly at random. Then

$$
F(T, \phi)=\inf _{\varepsilon>0} \limsup _{i \rightarrow \infty} \frac{1}{i} \log \left(\mathbb{E}\left[\left|\left\{\psi:\{1, \ldots, i\} \rightarrow A \mid d_{\sigma_{i}}^{*}(\phi, \psi) \leq \varepsilon\right\}\right|\right]\right) .
$$

This theorem is proven in Section 2. In Section 3 we deduce Theorem 1.3 from it. 
1.1. Application I: automorphism invariance. Let $G$ be a countable group or semigroup. Let $G \curvearrowright^{T}(X, \mu)$. Let $\omega: G \rightarrow G$ be an automorphism. Let $T^{\omega}=$ $\left(T_{g}^{\omega}\right)_{g \in G}$ where $T_{g}^{\omega} x:=T_{\omega(g)} x$ for all $x \in X$. This new action of $G$ is not necessarily isomorphic to the original action. That is, there might not exist a map $\phi: X \rightarrow X$ such that $\phi\left(T_{g} x\right)=T_{g}^{\omega} \phi(x)$ for a.e. $x \in X$ and all $g \in G$.

Suppose that $\Sigma=\left\{\sigma_{i}\right\}$ is an asymptotically free sequence of homomorphisms $\sigma_{i}: G \rightarrow \operatorname{Sym}\left(m_{i}\right)$. Let $\Sigma^{\omega}=\left\{\sigma_{i} \circ \omega\right\}$. A short exercise reveals that $h(\Sigma, T, \phi)=$ $h\left(\Sigma^{\omega}, T^{\omega}, \phi\right)$ for any $\phi$.

If $\sigma_{i}: G \rightarrow \operatorname{Sym}(i)$ is chosen uniformly at random, it follows that the law of $\sigma_{i} \circ \omega$ is the same as the law of $\sigma_{i}$. Therefore, if $\mu_{i}$ is the uniform probability measure on the set of homomorphisms from $G$ to $\operatorname{Sym}(i)$ and $\Sigma=\left\{\mu_{i}\right\}$, then $h(\Sigma, T, \phi)=h\left(\Sigma, T^{\omega}, \phi\right)$. Theorem 1.3 now implies:

Theorem 1.5. Let $G$ and $T$ be as in Theorem 1.3. Let $\omega: G \rightarrow G$ be an automorphism. Then $f(T, \phi)=f\left(T^{\omega}, \phi\right)$ for any finite observable $\phi$.

This implies that $f(T, \phi)$ does not depend on the choice of free generator set $\left\{s_{1}, \ldots, s_{r}\right\}$ for $G$ since any two free generating sets are related by an automorphism.

\subsection{Application II: lower bounds on the $f$-invariant of a factor}

Definition 5. Let $G \curvearrowright^{T}(X, \mu)$ and $G \curvearrowright^{S}(Y, v)$. Then $S$ is a factor of $T$ if there exists a measurable map $\phi: X \rightarrow Y$ such that $\phi_{*} \mu=v$ and $\phi\left(T_{g} x\right)=S_{g} \phi(x)$ for all $g \in G$ and a.e. $x \in X$.

To motivate this section, let us point out two curious facts.

First, Ornstein proved in [Or70] that every factor of a Bernoulli shift over $\mathbb{Z}$ is measurably conjugate to a Bernoulli shift. It is not known whether this holds when $\mathbb{Z}$ is replaced with a nonabelian free group. A counterexample due to Sorin Popa [Po08] (based on [PS07]) shows that if $G$ is an infinite property $T$ group then there exists a factor of a Bernoulli shift over $G$ that is not measurably conjugate to a Bernoulli shift.

Second, the $f$-invariant of an action can be negative. For example, if $X$ is a set with $n$ elements, $\mu$ is the uniform measure on $X$ and $T=\left(T_{g}\right)_{g \in G}$ is a measurepreserving action of $G=\left\langle s_{1}, \ldots, s_{r}\right\rangle$ on $X$ then $f(T)=-(r-1) \log (n)$.

From these two facts a natural question arises: can the $f$-invariant of a factor of a Bernoulli shift over $G$ be negative? To answer this, let us recall the following result from [Bo08b], Corollary 8.3.

Lemma 1.6. Let $G$ be a countable group. Let $\Sigma=\left\{\sigma_{i}\right\}_{i=1}^{\infty}$ be an asymptotically free sequence of homomorphisms $\sigma_{i}: G \rightarrow \operatorname{Sym}\left(m_{i}\right)$. Let $T$ be a measure-preserving action of $G$ and let $S$ be a factor of $T$. Assume that there exist finite-entropy 
generating partitions for $T$ and $S$. Also let $\phi$ be a generating observable for $T$ with $H(\phi)<\infty$. Then

$$
h(\Sigma, S) \geq h(\Sigma, T)-H(\phi)
$$

So Theorem 1.3 implies:

Theorem 1.7. Let $G=\left\langle s_{1}, \ldots, s_{r}\right\rangle$ be a free group on $r$ generators. Let $T$ be a measure-preserving action of $G$ and let $S$ be a factor of $T$. Assume there exists finite generating partitions for $T$ and $S$. Let $\alpha$ be a finite generating partition for $T$. Then

$$
f(S) \geq f(T)-H(\alpha)
$$

In order to apply this to Bernoulli shifts, let us recall the definitions. Let $K$ be a finite or countable set and $\kappa$ a probability measure on $K$. Let $\left(K^{G}, \kappa^{G}\right)$ denote the product measure space. Define $T_{g}: K^{G} \rightarrow K^{G}$ by $T_{g}(x)(h)=x(h g)$. This defines a measure-preserving action of $G$ on $\left(K^{G}, \kappa^{G}\right)$. It is the Bernoulli shift over $G$ with base measure $\kappa$. In [Bo08a] it was shown that $f(T)=H(\kappa)$ where

$$
H(\kappa):=-\sum_{k \in K} \mu(\{k\}) \log (\mu(\{k\}) .
$$

Let $\alpha$ be the canonical partition of $K^{G}$, i.e., $\alpha=\left\{A_{k}: k \in K\right\}$ where $A_{k}=$ $\left\{x \in K^{G} \mid x(e)=k\right\}$. Note $H(\alpha)=H(\kappa)=f(T)$. So the theorem above implies the following result.

Corollary 1.8. If $S$ is a factor of the Bernoulli shift and if there exists a finite generating partition for $S$ then $f(S) \geq 0$.

It is unknown whether there exists a nontrivial factor $S$ of a Bernoulli shift over a free group $G$ such that $f(S)=0$.

In [Bo08c], classical Markov chains are generalized to Markov chains over free groups. An explicit example was given of a Markov chain with finite negative $f$ invariant. It follows that this Markov chain cannot be measurably conjugate to a factor of a Bernoulli shift. It can be shown that this Markov chain is uniformly mixing. To contrast this with the classical case, recall that Friedman and Ornstein proved in [FO70] that every mixing Markov chain over the integers is isomorphic to a Bernoulli shift.

Now we can construct a mixing Markov chain with positive $f$-invariant that is not isomorphic to a Bernoulli shift as follows. Let $T$ denote a mixing Markov chain with negative $f$-invariant. Let $S$ denote a Bernoulli shift with $f(S)>-f(T)$. Consider the product action $T \times S$. A short computation reveals that, in general, $f(T \times S)=f(T)+f(S)$. Therefore $T \times S$ has positive $f$-invariant. It can be shown that $T \times S$ is a mixing Markov chain. However it cannot be isomorphic to a Bernoulli shift since it factors onto $T$ which has negative $f$-invariant. 


\section{Proof of Theorem 1.4}

Let $G=\left\langle s_{1}, \ldots, s_{r}\right\rangle$ be a free group or free semigroup of rank $r$. Let $G \curvearrowright^{T}(X, \mu)$. Let $\phi: X \rightarrow A$ be a finite observable.

We will need to consider certain perturbations of the measure $\mu$ with respect to the given observable $\phi: X \rightarrow A$. For this purpose we introduce the notion of weights on the graph $\mathscr{E}=(V, E)$ that is defined as follows. The vertex set $V$ equals $A$. For every $a, b \in A$ and every $i \in\{1, \ldots, r\}$ there is a directed edge from $a$ to $b$ labeled $i$. This edge is denoted $(a, b ; i)$. We allow the possibility that $a=b$. A weight on $\mathscr{G}$ is a function $W: V \sqcup E \rightarrow[0,1]$ satisfying

$$
\begin{aligned}
W(a) & =\sum_{b \in A} W(a, b ; i)=\sum_{b \in A} W(b, a ; i) \quad \text { for all } i=1 \ldots r, a \in A, \\
1 & =\sum_{a \in A} W(a) .
\end{aligned}
$$

For example,

$$
\begin{aligned}
W_{\mu}(a) & :=\mu\left(\phi^{-1}(a)\right), \\
W_{\mu}(a, b ; i) & :=\mu\left(\left\{x \in X \mid \phi(x)=a, \phi\left(T_{s_{i}} x\right)=b\right\}\right)
\end{aligned}
$$

is the weight associated to $\mu$. For a homomorphism $\sigma: G \rightarrow \operatorname{Sym}(n)$ and a function $\psi:\{1, \ldots, n\} \rightarrow A$ we define the weight $W_{\sigma, \psi}$ by

$$
\begin{aligned}
W_{\sigma, \psi}(a) & :=\left|\psi^{-1}(a)\right| / n, \\
W_{\sigma, \psi}(a, b ; i) & :=\left|\left\{j \mid \psi(j)=a, \psi\left(\sigma\left(s_{i}\right) j\right)=b\right\}\right| / n .
\end{aligned}
$$

Note that

$$
d_{\sigma}^{*}(\phi, \psi)=\sum_{i=1}^{r} \sum_{a, b \in A}\left|W_{\mu}(a, b ; i)-W_{\sigma, \psi}(a, b ; i)\right| .
$$

So given two weights $W_{1}, W_{2}$ define

$$
d_{*}\left(W_{1}, W_{2}\right):=\sum_{i=1}^{r} \sum_{a, b \in A}\left|W_{1}(a, b ; i)-W_{2}(a, b ; i)\right| .
$$

Proposition 2.1. Let $n$ be a positive integer. Let $W$ be a weight. Suppose that $W(a, b ; i) n \in \mathbb{Z}$ for every $a, b \in A$ and every $i=1 \ldots r$. If $\sigma: G \rightarrow \operatorname{Sym}(n)$ is chosen uniformly at random then

$$
\mathbb{E}\left[\left|\left\{\psi:\{1, \ldots, n\} \rightarrow A \mid d_{*}\left(W, W_{\sigma, \psi}\right)=0\right\}\right|\right]=\frac{n !^{1-r} \prod_{a \in A}(n W(a)) !^{2 r-1}}{\prod_{i=1}^{r} \prod_{a, b \in A}(n W(a, b ; i)) !} .
$$

Proof. Note that if $d_{*}\left(W, W_{\sigma, \psi}\right)=0$ then $W_{\sigma, \psi}(a)=W(a)$ for all $a \in A$. Equivalently,

$$
\left|\psi^{-1}(a)\right|=n W(a) \text { for all } a \in A .
$$


The number of functions $\psi:\{1, \ldots, n\} \rightarrow A$ that satisfy this requirement is

$$
\frac{n !}{\prod_{a \in A}(n W(a)) !} \text {. }
$$

If $\psi_{1}, \psi_{2}$ are two different functions that satisfy equation (1) then there is a permutation $\tau \in \operatorname{Sym}(n)$ such that $\psi_{1}=\psi_{2} \circ \tau$. If $\sigma^{\tau}: G \rightarrow \operatorname{Sym}(n)$ is the homomorphism defined by $\sigma^{\tau}(g)=\tau \sigma(g) \tau^{-1}$ then $W_{\sigma, \psi_{1}}=W_{\sigma^{\tau}, \psi_{2}}$. Since $\sigma: G \rightarrow \operatorname{Sym}(n)$ is chosen uniformly at random, this implies that the probability that $d_{*}\left(W, W_{\sigma, \psi_{1}}\right)=0$ is the same as the probability that $d_{*}\left(W, W_{\sigma, \psi_{2}}\right)=0$. So fix a particular function $\psi_{0}$ satisfying equation (1). Then

$$
\mathbb{E}\left[\left|\left\{\psi:\{1, \ldots, n\} \rightarrow A \mid d_{*}\left(W, W_{\sigma, \psi}\right)=0\right\}\right|\right]=\frac{n ! \operatorname{Prob}\left[d_{*}\left(W, W_{\sigma, \psi_{0}}\right)=0\right]}{\prod_{a \in A}(n W(a)) !} .
$$

For any two weights $W_{1}, W_{2}$ and $1 \leq i \leq r$, define

$$
d_{i}\left(W_{1}, W_{2}\right):=\sum_{a, b \in A}\left|W_{1}(a, b ; i)-W_{2}(a, b ; i)\right| .
$$

So $d_{*}=\sum_{i=1}^{r} d_{i}$.

The homomorphism $\sigma: G \rightarrow \operatorname{Sym}(n)$ is determined by its values $\sigma\left(s_{1}\right), \ldots, \sigma\left(s_{r}\right)$. The event $d_{i}\left(W, W_{\sigma, \psi_{0}}\right)=0$ is determined by $\sigma\left(s_{i}\right)$. So if $i \neq j$ then the events $d_{i}\left(W, W_{\sigma, \psi_{0}}\right)=0$ and $d_{j}\left(W, W_{\sigma, \psi_{0}}\right)=0$ are independent. Therefore,

$$
\begin{gathered}
\mathbb{E}\left[\left|\left\{\psi:\{1, \ldots, n\} \rightarrow A \mid d_{*}\left(W, W_{\sigma, \psi}\right)=0\right\}\right|\right] \\
=\frac{n ! \prod_{i=1}^{r} \operatorname{Prob}\left[d_{i}\left(W, W_{\sigma, \psi_{0}}\right)=0\right]}{\prod_{a \in A}(n W(a)) !} .
\end{gathered}
$$

Fix $i \in\{1, \ldots, r\}$. We will compute $\operatorname{Prob}\left[d_{i}\left(W, W_{\sigma, \psi_{0}}\right)=0\right]$. The element $\sigma\left(s_{i}\right)$ induces a pair of partitions $\alpha, \beta$ of $\{1, \ldots, n\}$ as follows: $\alpha:=\left\{P_{a, b} \mid a, b \in A\right\}$ and $\beta:=\left\{Q_{a, b} \mid a, b \in A\right\}$, where

$$
\begin{aligned}
P_{a, b} & =\left\{j \mid \psi_{0}(j)=a \text { and } \psi_{0}\left(\sigma\left(s_{i}\right) j\right)=b\right\}, \\
Q_{a, b} & =\left\{j \mid \psi_{0}(j)=b \text { and } \psi_{0}\left(\sigma\left(s_{i}\right)^{-1} j\right)=a\right\} .
\end{aligned}
$$

Also there is a bijection from $M_{a, b}: P_{a, b} \rightarrow Q_{a, b}$ defined by $M_{a, b}(j)=\sigma\left(s_{i}\right) j$. Conversely, $\sigma\left(s_{i}\right)$ is uniquely determined by these partitions and bijections.

Note that $\left|P_{a, b}\right|=\left|Q_{a, b}\right|=n W_{\sigma, \psi_{0}}(a, b ; i)$. Thus $d_{i}\left(W, W_{\sigma, \psi_{0}}\right)=0$ if and only $\left|P_{a, b}\right|=\left|Q_{a, b}\right|=n W(a, b ; i)$ for all $a, b \in A$. If this occurs then $\left|\bigcup_{b \in A} P_{a, b}\right|=$ $n W(a)$ for all $a \in A$. So the number of pairs of partitions $\alpha, \beta$ that satisfy this requirement is

$$
\frac{\prod_{a \in A}(n W(a)) !^{2}}{\left.\prod_{a, b \in A}(n W(a, b ; i)) !\right)^{2}} .
$$


Given such a pair of partitions, the number of collections of bijections

$$
M_{a, b}: P_{a, b} \rightarrow Q_{a, b}
$$

(for $a, b \in A)$ equals $\prod_{a, b \in A}(n W(a, b ; i))$ !. Since there are $n$ ! elements in $\operatorname{Sym}(n)$ it follows that

$$
\operatorname{Prob}\left[d_{i}\left(W, W_{\sigma, \psi_{0}}\right)=0\right]=\frac{\prod_{a \in A}(n W(a)) !^{2}}{n ! \prod_{a, b \in A}(n W(a, b ; i)) !} .
$$

The proposition now follows from this equality and equation (2).

Let $\mathcal{W}$ be the set of all weights on $\mathcal{E}$. It is a compact convex subset of $\mathbb{R}^{d}$ for some $d>0$. Define $F: \mathcal{W} \rightarrow \mathbb{R}$ by

$$
F(W):=-\left(\sum_{i=1}^{r} \sum_{a, b \in A} W(a, b ; i) \log (W(a, b ; i))\right)+(2 r-1) \sum_{a \in A} W(a) \log (W(a)) .
$$

We follow the usual convention that $0 \log (0)=0$. Observe that $F(T, \phi)=F\left(W_{\mu}\right)$.

Given a weight $W$, let $\mathrm{q}_{W}$ denote the smallest positive integer such that $W(a, b ; i) \mathrm{q}_{W} \in \mathbb{Z}$ for all $a, b \in A$ and for all $i \in\{1, \ldots, r\}$. If no such integer exists then set $\mathrm{q}_{W}:=+\infty$. If $p$ and $q$ are integers, $p \neq 0$ and $\frac{q}{p} \in \mathbb{Z}$ then we write $p \mid q$. Otherwise we write $p \nmid q$.

Lemma 2.2. $F: \mathcal{W} \rightarrow \mathbb{R}$ is continuous. Also, there exist constants $0<c_{1}<c_{2}$ and $p_{1}<p_{2}$ such that for every weight $W$ with $\mathrm{q}_{W}<\infty$ and every $n \geq 1$ such that $\mathrm{q}_{W} \mid n$, if $\sigma: G \rightarrow \operatorname{Sym}(n)$ is chosen uniformly at random then

$$
c_{1} n^{p_{1}} e^{F(W) n} \leq \mathbb{E}\left[\left|\left\{\psi:\{1, \ldots, n\} \rightarrow A \mid d_{*}\left(W, W_{\sigma, \psi}\right)=0\right\}\right|\right] \leq c_{2} n^{p_{2}} e^{F(W) n} .
$$

Proof. It is obvious that $F$ is continuous. The second statement follows from the previous proposition and Stirling's approximation. The constants depend only on $|A|$ and the rank $r$ of $G$.

Lemma 2.3. There exists a constant $k>0$ such that the following holds. Let $W$ be $a$ weight and let $n>0$ be a positive integer. Then there exists a weight $\widetilde{W}$ such that $\mathrm{q}_{\widetilde{W}}<\infty, \mathrm{q}_{\widetilde{W}} \mid n$ and $d_{*}(W, \widetilde{W})<k / n$.

Proof. Choose $a_{0} \in A$. For $b, c \in A-\left\{a_{0}\right\}$ and $i \in\{1, \ldots, r\}$ define

$$
\begin{aligned}
\widetilde{W}(b) & :=\frac{\lfloor W(b) n\rfloor}{n}, \\
\widetilde{W}\left(a_{0}\right) & :=1-\sum_{b \in A-\left\{a_{0}\right\}} \widetilde{W}(b),
\end{aligned}
$$




$$
\begin{aligned}
\widetilde{W}(b, c ; i) & :=\frac{\lfloor W(b, c ; i) n\rfloor}{n}, \\
\widetilde{W}\left(a_{0}, b ; i\right) & :=\widetilde{W}(b)-\sum_{a \in A-\left\{a_{0}\right\}} \widetilde{W}(a, b ; i), \\
\widetilde{W}\left(b, a_{0} ; i\right) & :=\widetilde{W}(b)-\sum_{a \in A-\left\{a_{0}\right\}} \widetilde{W}(b, a ; i), \\
\widetilde{W}\left(a_{0}, a_{0} ; i\right) & :=\widetilde{W}\left(a_{0}\right)-\sum_{b \in A-\left\{a_{0}\right\}} \widetilde{W}\left(a_{0}, b ; i\right) .
\end{aligned}
$$

Let us check that $\widetilde{W}$ is a weight. It is clear that $\sum_{a \in A} \widetilde{W}(a)=1$. If $b \in A-\left\{a_{0}\right\}$ then $\widetilde{W}(b)=\sum_{a \in A} \widetilde{W}(a, b ; i)=\sum_{a \in A} \widetilde{W}(b, a ; i)$. It is immediate that $\widetilde{W}\left(a_{0}\right)=$ $\sum_{b \in A} \widetilde{W}\left(a_{0}, b ; i\right)$. Also

$$
\begin{aligned}
\sum_{b \in A} \widetilde{W}\left(b, a_{0} ; i\right) & =\widetilde{W}\left(a_{0}, a_{0} ; i\right)+\sum_{b \in A-\left\{a_{0}\right\}} \widetilde{W}\left(b, a_{0} ; i\right) \\
& =\widetilde{W}\left(a_{0}\right)-\sum_{b \in A-\left\{a_{0}\right\}} \widetilde{W}\left(a_{0}, b ; i\right)+\sum_{b \in A-\left\{a_{0}\right\}} \widetilde{W}\left(b, a_{0} ; i\right) \\
& =\widetilde{W}\left(a_{0}\right)+\sum_{b \in A-\left\{a_{0}\right\}} \widetilde{W}\left(b, a_{0} ; i\right)-\widetilde{W}\left(a_{0}, b ; i\right) \\
& =\widetilde{W}\left(a_{0}\right)+\sum_{b \in A-\left\{a_{0}\right\}}(\widetilde{W}(b) \\
& \left.-\sum_{a \in A-\left\{a_{0}\right\}} \widetilde{W}(b, a ; i)\right)-\left(\widetilde{W}(b)-\sum_{a \in A-\left\{a_{0}\right\}} \widetilde{W}(a, b ; i)\right) \\
& =\widetilde{W}\left(a_{0}\right) .
\end{aligned}
$$

This proves that $\widetilde{W}$ is a weight. It is clear that $\mathrm{q}_{\widetilde{W}}<\infty$ and $\mathrm{q}_{\widetilde{W}} \mid n$. Lastly observe that if $a, b \in A-\left\{a_{0}\right\}$ then $|W(a, b ; i)-\widetilde{W}(a, b ; i)| \leq 1 / n$. Since $|W(b)-\widetilde{W}(b)| \leq 1 / n$ too, $\left|W\left(a_{0}, b ; i\right)-\widetilde{W}\left(a_{0}, b ; i\right)\right| \leq|A| / n$ and $\left|\bar{W}\left(b, a_{0} ; i\right)-\widetilde{W}\left(b, a_{0} ; i\right)\right| \leq|A| / n$. Since $\left|W\left(a_{0}\right)-\widetilde{W}\left(a_{0}\right)\right| \leq|A| / \bar{n},\left|W\left(a_{0}, a_{0} ; i\right)-\widetilde{W}\left(a_{0}, a_{0} ; i\right)\right| \leq|A|^{2} / n$. Thus $d_{*}(W, \widetilde{W}) \leq r|A|^{2} / n$.

We are now ready to prove Theorem 1.4.

Proof of Theorem 1.4. Recall that $\phi: X \rightarrow A$ is an observable and $A$ is a finite set. Let $n \geq 0$ and let $\sigma_{n}: G \rightarrow \operatorname{Sym}(n)$ be a homomorphism chosen uniformly at random. Given a weight $W$, let

$$
Z_{n}(W):=\left|\left\{\psi:\{1, \ldots, n\} \rightarrow A \mid d_{*}\left(W_{\sigma_{n}, \psi}, W\right)=0\right\}\right| .
$$

For any $\varepsilon>0$,

$$
\mathbb{E}\left[\left|\left\{\psi:\{1, \ldots, n\} \rightarrow A \mid d_{\sigma_{n}}^{*}(\phi, \psi) \leq \varepsilon\right\}\right|\right]=\sum_{W: d_{*}\left(W, W_{\mu}\right) \leq \varepsilon} \mathbb{E}\left[Z_{n}(W)\right] .
$$


Let $\delta>0$. Since $F: \mathcal{W} \rightarrow \mathbb{R}$ is continuous, there exists $\varepsilon_{0}>0$ such that if $d_{*}\left(W, W_{\mu}\right) \leq \varepsilon_{0}$ then $\left|F(W)-F\left(W_{\mu}\right)\right|<\delta$. So let us fix $\varepsilon$ with $0<\varepsilon<\varepsilon_{0}$.

By the previous lemma, if $n$ is sufficiently large then there exists a weight $W$ such that $d_{*}\left(W, W_{\mu}\right) \leq \varepsilon$ and $\mathrm{q}_{W} \mid n$. Lemma 2.2 implies

$$
\mathbb{E}\left[\left|\left\{\psi:\{1, \ldots, n\} \rightarrow A \mid d_{\sigma_{n}}^{*}(\phi, \psi) \leq \varepsilon\right\}\right|\right] \geq \mathbb{E}\left[Z_{n}(W)\right] \geq c_{1} n^{p_{1}} e^{F\left(W_{\mu}\right) n-\delta n},
$$

where $c_{1}>0$ and $p_{1}$ are constants.

If $W$ is a weight such that $\mathrm{q}_{W} \nmid n$ then $Z_{n}(W)=0$. If $\mathrm{q}_{W} \mid n$ then $W(a, b ; i) \in$ $\mathbb{Z}[1 / n]$ for all $a, b \in A$ and $i \in\{1, \ldots, r\}$. The space of all weights lies inside the cube $[0,1]^{d} \subset \mathbb{R}^{d}$ for some $d$. So the number of weights $W$ such that $Z_{n}(W) \neq 0$ is at most $n^{d}$. Lemma 2.2 and equation (3) now imply that

$$
\mathbb{E}\left[\left|\left\{\psi:\{1, \ldots, n\} \rightarrow A \mid d_{\sigma_{n}}^{*}(\phi, \psi) \leq \varepsilon\right\}\right|\right] \leq c_{2} n^{p_{2}+d} e^{F\left(W_{\mu}\right) n+\delta n} .
$$

Here $c_{2}>0$ and $p_{2}$ are constants. Equations (4) and (5) imply

$$
\limsup _{n \rightarrow \infty}\left|\frac{1}{n} \log \left(\mathbb{E}\left[\left|\left\{\psi:\{1, \ldots, n\} \rightarrow A \mid d_{\sigma_{n}}^{*}(\phi, \psi) \leq \varepsilon\right\}\right|\right]\right)-F\left(W_{\mu}\right)\right| \leq \delta .
$$

Since $\delta$ is arbitrary, it follows that

$$
\inf _{\varepsilon>0} \lim _{n \rightarrow \infty} \frac{1}{n} \log \left(\mathbb{E}\left[\left|\left\{\psi:\{1, \ldots, n\} \rightarrow A \mid d_{\sigma_{n}}^{*}(\phi, \psi) \leq \varepsilon\right\}\right|\right]\right)=F\left(W_{\mu}\right)=F(T, \phi) .
$$

\section{Proof of Theorem 1.3}

As in the statement of Theorem 1.3, let $G=\left\langle s_{1}, \ldots, s_{r}\right\rangle$ be a free group or free semigroup of rank $r \geq 1$. Let $G \curvearrowright^{T}(X, \mu)$. Let $\phi: X \rightarrow A$ be a finite observable. Let $\Sigma=\left\{\mu_{i}\right\}_{i=1}^{\infty}$ where each $\mu_{i}$ is the uniform probability measure on the set of homomorphisms from $G$ to $\operatorname{Sym}(i)$. Let $\sigma_{i}: G \rightarrow \operatorname{Sym}(i)$ be a homomorphism chosen uniformly at random among all homomorphisms of $G$ into $\operatorname{Sym}(i)$. Theorem 1.3 is an immediate consequence of the next two propositions.

Proposition 3.1. $h(\Sigma, T, \phi) \leq f(T, \phi)$.

Proof. Let $S=\left\{e, s_{1}, \ldots, s_{r}\right\}$. Observe that for any $n$, if $\psi:\{1, \ldots, n\} \rightarrow A$ is any function then $d_{\sigma_{n}}^{S}(\phi, \psi) r \geq d_{\sigma_{n}}^{*}(\phi, \psi)$. So if $\varepsilon>0$ then

$$
\begin{aligned}
\mathbb{E}\left[\left|\left\{\psi:\{1, \ldots, n\} \rightarrow A \mid d_{\sigma_{n}}^{S}(\phi, \psi) \leq \varepsilon\right\}\right|\right] \\
\quad \leq \mathbb{E}\left[\left|\left\{\psi:\{1, \ldots, n\} \rightarrow A \mid d_{\sigma_{n}}^{*}(\phi, \psi) \leq r \varepsilon\right\}\right|\right] .
\end{aligned}
$$

This implies $h(\Sigma, T, \phi) \leq F(T, \phi)$. 
Recall that $B(e, n)$ denotes the ball of radius $n$ in $G$. Furthermore we have $f(T, \phi)=\inf _{n} F\left(T, \phi^{B(e, n)}\right)$, and thus $\inf _{n} h\left(\Sigma, T, \phi^{B(e, n)}\right) \leq f(T, \phi)$. Since $\phi$ and $\phi^{B(e, n)}$ generate the same $\sigma$-algebra, Theorem 1.1 implies that $h(\Sigma, T, \phi)=$ $h\left(\Sigma, T, \phi^{B(e, n)}\right)$ for all $n$. This implies the proposition.

Proposition 3.2. $h(\Sigma, T, \phi) \geq f(T, \phi)$.

Proof. Given a finite set $K \subset G$, define

$$
h(\Sigma, T, \phi ; K):=\inf _{\varepsilon>0} \limsup _{n \rightarrow \infty} \frac{1}{n} \log \left(\mathbb{E}\left[\left|\left\{\psi:\{1, \ldots, n\} \rightarrow A \mid d_{\sigma_{n}}^{K}(\phi, \psi) \leq \varepsilon\right\}\right|\right]\right) .
$$

Claim 1. $h(\Sigma, T, \phi ; B(e, m)) \geq F\left(T, \phi^{B(e, m)}\right)$ for all $m \geq 0$.

Note that if $K \subset L$ then $h(\Sigma, T, \phi ; K) \geq h(\Sigma, T, \phi ; L)$ holds. It follows that $h(\Sigma, T, \phi)=\inf _{m} h(\Sigma, T, \phi ; B(e, m))$. Thus claim 1 implies the proposition.

To simplify notation, let $\mathrm{B}$ denote $B(e, m)$. To prove claim 1 , for $m, n, \varepsilon \geq 0$, let $P(m, n, \varepsilon)$ be the set of all pairs $(\sigma, \omega)$ with $\sigma: G \rightarrow \operatorname{Sym}(n)$ a homomorphism and $\omega:\{1, \ldots, n\} \rightarrow A$ a map such that $d_{\sigma}^{\mathrm{B}}(\phi, \omega) \leq \varepsilon$. Since there are $n !^{r}$ homomorphisms from $G$ into $\operatorname{Sym}(n)$,

$$
h(\Sigma, T, \phi ; \mathrm{B})=\inf _{\varepsilon>0} \limsup _{n \rightarrow \infty} \frac{1}{n} \log \left(\frac{|P(m, n, \varepsilon)|}{n !^{r}}\right) .
$$

Let $Q(m, n, \varepsilon)$ be the set of all pairs $(\sigma, \psi)$ with $\sigma: G \rightarrow \operatorname{Sym}(n)$ a homomorphism and $\psi:\{1, \ldots, n\} \rightarrow A^{\mathrm{B}}$ a map such that $d_{\sigma}^{*}\left(\phi^{\mathrm{B}}, \psi\right) \leq \varepsilon$. By Theorem 1.4,

$$
F\left(T, \phi^{\mathrm{B}}\right)=\inf _{\varepsilon>0} \limsup _{n \rightarrow \infty} \frac{1}{n} \log \left(\frac{|Q(m, n, \varepsilon)|}{n !^{r}}\right) .
$$

For $g \in \mathrm{B}$ let $\pi_{g}: A^{\mathrm{B}} \rightarrow A$ denote the projection map $\pi_{g}\left(\left(a_{h}\right)_{h \in \mathrm{B}}\right)=a_{g}$. For $(\sigma, \psi) \in Q(m, n, \varepsilon)$, define $R(\sigma, \psi)=\left(\sigma, \pi_{e} \circ \psi\right)$. Define $H(x):=-x \log (x)-$ $(1-x) \log (1-x)$.

Claim 2. If $c=1+|\mathrm{B}|$ then the image of $R$ is contained in $P(m, n, \varepsilon c)$.

Claim 3. There are constants $C, k>0$ depending only on $m$ such that if $\varepsilon<\frac{1}{4|B|}$ then $R$ is at most $C \exp (n k \varepsilon+n H(2|\mathrm{~B}| \varepsilon))$ to 1, i.e., for any $(\sigma, \omega)$ in the image of $R,\left|R^{-1}(\sigma, \omega)\right| \leq C \exp (n k \varepsilon+n H(2|\mathrm{~B}| \varepsilon))$.

Claims 2 and 3 imply

$$
C \exp (k n \varepsilon+n H(2|\mathrm{~B}| \varepsilon)) \mid P(m, n, \varepsilon c))|\geq| Q(m, n, \varepsilon) \mid .
$$

Together with equations (6) and (7), this implies claim 1 and hence the proposition.

Next we prove claim 2. For this purpose, fix a homomorphism $\sigma: G \rightarrow \operatorname{Sym}(n)$. Observe that for any $x \in X$ and any $t \in\left\{s_{1}, \ldots, s_{r}\right\}$,

$$
\pi_{g} \phi^{\mathrm{B}}(x)=\phi\left(T_{g} x\right)=\pi_{g t^{-1}} \phi^{\mathrm{B}}\left(T_{t} x\right) \quad \text { for all } g \in \mathrm{B} \cap \mathrm{B} t .
$$


Therefore if $i \in\{1, \ldots, n\}$ and, for some $g \in \mathrm{B} \cap \mathrm{B} t, \psi:\{1, \ldots, n\} \rightarrow A^{\mathrm{B}}$ satisfies

$$
\pi_{g} \psi(i) \neq \pi_{g t^{-1}} \psi(\sigma(t) i),
$$

then $\psi \vee \psi^{t}(i) \neq \phi^{\mathrm{B}} \vee \phi^{\mathrm{B} t}(x)$ for any $x \in X$.

So let $\mathcal{G}$ be the set of all $i \in\{1, \ldots, n\}$ such that for all $t \in\left\{s_{1}, \ldots, s_{r}\right\}$,

$$
\pi_{g} \psi(i)=\pi_{g t^{-1}} \psi(\sigma(t) i) \quad \text { for all } g \in \mathrm{B} \cap \mathrm{B} t \text {. }
$$

Thus

$$
d_{\sigma}^{*}\left(\phi^{\mathrm{B}}, \psi\right) \geq \frac{\left|\mathscr{E}^{c}\right|}{n}=\zeta\left(\mathscr{E}^{c}\right)
$$

where $\mathscr{G}^{c}$ denotes the complement of $\mathcal{E}$ and $\zeta$ denotes the uniform probability measure on $\{1, \ldots, n\}$.

Let $\mathscr{S}_{m}$ be the set of all $i \in\{1, \ldots, n\}$ such that $\sigma(g) i \in \mathcal{G}$ for all $g \in$ B. Note that

$$
\zeta\left(\mathscr{E}_{m}^{c}\right) \leq|\mathrm{B}| \zeta\left(\mathscr{E}^{c}\right) \leq|\mathrm{B}| d_{\sigma}^{*}\left(\phi^{\mathrm{B}}, \psi\right) .
$$

If $i \in \mathscr{S}_{m}$ then $\psi(i)=\left(\pi_{e} \circ \psi\right)^{\mathrm{B}}(i)$. Therefore

$$
\begin{aligned}
\sum_{a \in A^{\mathrm{B}}}\left|\psi_{*} \zeta(a)-\left(\pi_{e} \circ \psi\right)_{*}^{\mathrm{B}} \zeta(a)\right| & \leq \frac{1}{n}\left|\left\{i \mid \psi(i) \neq\left(\pi_{e} \circ \psi\right)^{\mathrm{B}}(i)\right\}\right| \\
& \leq \zeta\left(\mathscr{G}_{m}^{c}\right) \leq|\mathrm{B}| d_{\sigma}^{*}\left(\phi^{\mathrm{B}}, \psi\right) .
\end{aligned}
$$

Suppose that $d_{\sigma}^{*}\left(\phi^{\mathrm{B}}, \psi\right) \leq \varepsilon$. Then

$$
\begin{aligned}
d_{\sigma}^{\mathrm{B}}\left(\phi, \pi_{e} \circ \psi\right) & =\sum_{a \in A^{\mathrm{B}}}\left|\phi_{*}^{\mathrm{B}} \mu(a)-\left(\pi_{e} \circ \psi\right)_{*}^{\mathrm{B}} \zeta(a)\right| \\
& \leq \sum_{a \in A^{\mathrm{B}}}\left|\phi_{*}^{\mathrm{B}} \mu(a)-\psi_{*} \zeta(a)\right|+\left|\psi_{*} \zeta(a)-\left(\pi_{e} \circ \psi\right)_{*}^{\mathrm{B}} \zeta(a)\right| \\
& \leq d_{\sigma}^{*}\left(\phi^{\mathrm{B}}, \psi\right)(1+|\mathrm{B}|) \leq \varepsilon(1+|\mathrm{B}|) .
\end{aligned}
$$

This proves claim 2 .

Let $(\sigma, \omega)$ be in the image of $R$.

Claim 4. For every $\psi$ with $R(\sigma, \psi)=(\sigma, \omega)$, there exists a set $L(\psi) \subset\{1, \ldots, n\}$ of cardinality $\lfloor n(1-|\mathrm{B}| \varepsilon)\rfloor$ such that $\psi(i)=\omega^{B}(i)$ for all $i \in L(\psi)$.

To prove claim 4 , observe that if $\mathscr{E}_{m}$ is defined as above, then $\psi(i)=\omega^{B}(i)$ for all $i \in \mathscr{G}_{m}$. By equation (8),

$$
\left|\mathscr{E}_{m}\right|=n\left(1-\zeta\left(\mathscr{G}_{m}^{c}\right)\right) \geq n\left(1-|B| d_{\sigma}^{*}\left(\phi^{B}, \psi\right)\right) \geq n(1-|B| \varepsilon) .
$$

So let $L(\psi)$ be any subset of $\mathscr{S}_{m}$ with cardinality $\lfloor n(1-|\mathrm{B}| \varepsilon)\rfloor$. This proves claim 4 .

Next we prove claim 3. Claim 4 implies

$$
\left|R^{-1}(\sigma, \omega)\right| \leq|A|^{|\mathrm{B}|(n-\lfloor n(1-|\mathrm{B}| \varepsilon)\rfloor)}\left(\begin{array}{c}
n \\
\lfloor n(1-|\mathrm{B}| \varepsilon)\rfloor
\end{array}\right) .
$$


This is because there are $\left(\begin{array}{c}n \\ \lfloor n(1-|\mathrm{B}| \varepsilon)\rfloor\end{array}\right)$ sets in $\{1, \ldots, n\}$ with cardinality equal to $\lfloor n(1-|\mathrm{B}| \varepsilon)\rfloor$ and for each $i \in\{1, \ldots, n\}-L(\psi)$, there are at most $|A|^{|\mathrm{B}|}$ possible values for $\psi(i)$.

Because $H$ is monotone increasing for $0<x<1 / 2$ it follows from Stirling's approximation that if $\varepsilon<\frac{1}{4|\mathrm{~B}|}$ then

$$
\left(\begin{array}{c}
n \\
\lfloor n(1-|\mathrm{B}| \varepsilon)\rfloor
\end{array}\right) \leq C \exp (n H(2|\mathrm{~B}| \varepsilon)),
$$

where $C>0$ is a constant. This and equation (9) now imply claim 3 and hence the proposition.

\section{References}

[Bo08a] L. Bowen, A measure-conjugacy invariant for actions of free groups. Ann. of Math, to appear.

[Bo08b] L. Bowen, Measure conjugacy invariants for actions of countable sofic groups. $J$. Amer. Math. Soc. 23 (2010), 217-245. Zbl MR 2552252

[Bo08c] L. Bowen, Nonabelian free group actions: Markov processes, the Abramov-Rohlin formula and Yuzvinskii's formula. Preprint 2008. arXiv:0806.4420

[FO70] N. A. Friedman and D. S. Ornstein, On isomorphism of weak Bernoulli transformations. Adv. Math. 5 (1970), 365-394. Zbl 0203.05801 MR 0274718

[Or70] D. Ornstein, Factors of Bernoulli shifts are Bernoulli shifts. Adv. Math. 5 (1970), 349-364. Zbl 0227.28015 MR 0274717

[Po08] S. Popa, Private communication.

[PS07] S. Popa and R. Sasyk, On the cohomology of Bernoulli actions. Ergodic Theory Dynam. Systems 27 (2007), 241-251. Zbl 05144564 MR 2297095

Received March 11, 2009; revised June 24, 2009

L. Bowen, University of Hawai'i, Mānoa, 2565 McCarthy Mall, Keller 401A, Honolulu, Hawaii 96822, U.S.A., and Mathematics Department, MS-3368, Texas A\&M University, College Station, TX 77843-3368, U.S.A.

E-mail: lpbowen@math.tamu.edu 